\title{
Superior Reconstruction Quality Improvement of CT Image for Bias Correction Variance Measures
}

\author{
S.Asif Hussain \\ Assistant professor \\ Department of ECE, A.I.T.S, \\ Rajampet, Andhra Pradesh
}

\author{
M.N.Giri Prasad,PhD. \\ Professor, Department of \\ E.C.E., J.N.T.U.A, \\ Anantapur, Andhra Pradesh, \\ India
}

\author{
D. Satyanarayana, PhD. \\ Professor, Department of \\ ECE,RGMCET \\ Nandyal, A.P
}

\begin{abstract}
Image segmentation algorithms based on ROI typically rely on the homogeneity of image intensities. CT scanner is dedicated as research Scanner which has been developed in view of imaging applications. A key Feature of the work is to use Empirical system to achieve resolution recovery with novel region based method. This method identifies local intensity cluster with local clustering criterion function with respect to neighborhood center. Reconstruction quality is analyzed quantitatively in terms bias field correction for intensity inhomogenity correction. This method is valid on synthetic images of various imaging modalities. A significant improvement in reconstruction quality can be realized by faster and more accurate visual quality quantitative measures where Reconstruction quality is analyzed quantitatively in terms of bias-variance measures (bar phantom) and mean square error (lesion phantom). However, with the inclusion of the empirical kernel, the iterative algorithms provide superior reconstructions compared to FBP, both in terms of visual quality and quantitative measures. Simulated results show improved tumor bias and variance characteristics with the proposed algorithm.
\end{abstract}

\section{Keywords}

Intensity inhomogeneities, Empirical system kernel, Biasvariance, Iterative algorithms.

\section{INTRODUCTION}

Image segmentation is often an essential step before further image-processing of three-dimensional medical images can be done[23]. An object can be segmented based on shape and/or intensity characteristics. The task of image segmentation can be simplified with initialized parameters to guide accurate segmentation. Semi-automated and interactive methods [1] have been relatively successful, but require varying degrees of human input. Segmentation is often an important step in US B-mode image analysis .we consider the problem of correcting for attenuation-related intensity inhomogenieties i.e., those that cause a slowly changing (low-frequency) intensity contrast and are not due to speckle-mode imaging artifacts include speckle noise, attenuation(absorption and scattering), etc. The statistical analysis and reduction of speckle noise has been studied extensively in the literature [2]-[8]. Other artifacts, particularly those caused by non uniform beam attenuation within the body that are not accounted for by time gain compensation (TGC), also decrease the image signal-to-noise ratio (SNR).Existing level set methods for image segmentation can be categorized into two major classes: region-based models [9], [10],[15], [16], [18], [20] and edge-based models [11], [12], [13], [14],[19]. Region-based models aim to identify each region of interest by using a certain region descriptor to guide the motion of the active contour. However, it is very difficult to define a region descriptor for images with intensity in homogeneities. Most of region-based models [9], [21]-[12] are based on the assumption of intensity homogeneity. Edge-based models use edge information for image segmentation. These models do not assume homogeneity of image intensities, and thus can be applied to images with intensity in homogeneities. A novel region-based method for image segmentation. From a generally accepted model of images with intensity inhomogeneities, we derive a local intensity clustering property, and therefore define a local clustering criterion function for the intensities in a neighbourhood of each point. This local clustering criterion is integrated over the neighbourhood center to define an energy functional, which is converted to a level set formulation. Minimization of this energy is achieved by an interleaved process of level set evolution and estimation of the bias field. As an important application, our method can be used for segmentation and bias correction of magnetic resonance(MR) images.

\section{METHODS}

\subsection{Background}

In this section, we review the method proposed by Zhang for estimating the field $\mathrm{B}_{1}$ distortion and simultaneously segmenting an MR image and provide implementation details on how it has been adapted to work with US images. This method essentially estimates the low (spatial)-frequency multiplicative degradation field while at the same time identifying regions of similar intensity inhomogeneity using an MRF-MAP frame work. As we will explain in Section III, although developed for another imaging modality, under simplified assumptions, we can justify using the same approach on displayed US images.

\subsection{Model specification}

Let $\mathrm{S}$ be a lattice indexing the pixels in the given image. Further,

$$
I=\left(I_{1, \ldots,} I_{N) \text { and }} I^{*}=\left(I_{1}^{*}, \ldots, I_{N}^{*}\right)\right.
$$

let and be the observed and the ideal (that is, without intensity inhomogeneity distortion) intensities of the given image respectively, being the number of pixels in the image. We assume that the distortion at pixel $1 \leq i \leq N$ can be expressed by a multiplicative model of the form

$$
\mathrm{I}_{\mathrm{i}=} \mathrm{I}_{\mathrm{i}}^{*} \times \mathrm{d}_{\mathrm{i}}(1)
$$

Where $d_{i}$ represents the gain of the intensity due to the intensity inhomogeneity at pixel. A logarithmic 
transformation of this equation yields an addition. Let $y$ and $y^{*}$ denote, respectively, the observed and the ideal logtransformed intensities, then

$$
y=y^{*}+d
$$

Where $d$ denotes the log-transformed intensity distortion field. Segmentation can be considered as a problem of statistical classification, which is to assign every pixel a class label from a label set. Let $L$ denote the label set. A labeling of $S$ will be denoted by $X$, in which $x_{i} x_{i} \in L$ is the corresponding class label of pixel $i$. Given the class label $x_{i}$, it is assumed that the intensity value at pixel $i, y_{i}^{*}$ follows a Gaussian distribution(this assumption will be justified in Section III) with parameter $\theta\left(x_{i}\right)=\left(\mu_{x i}, \sigma_{x i}\right), \mu_{x i}, \sigma_{x i}$ being the mean and the variance of class $x_{i}$, respectively

$$
\mathrm{p}\left(\mathrm{y}_{\mathrm{i}}^{*} \mid \mathrm{x}_{\mathrm{i}}\right)=\mathrm{g}\left(\mathrm{y}_{\mathrm{i}}^{*} ; \theta\left(\mathrm{x}_{\mathrm{i}}\right)\right)(3)
$$

where

$$
g(y ; \theta)=1 / \sqrt{2 \pi \sigma^{2}} \exp \left(-\frac{\left.(y-\mu)^{2}\right)}{2 \sigma^{2}}\right) .
$$

With the distortion field taken into account, the above distribution can be written in terms of the observed intensity $y_{i}$ as

$$
p\left(y_{i} \mid x_{i}, d_{i}\right)=g\left(y_{i}-d_{i} ; \theta\left(x_{i}\right)(4)\right.
$$

and, hence, a class-independent intensity distribution

$$
p\left(y_{i} \mid d_{i}\right)=\sum_{j \in L}\left\{g\left(y_{i}-d_{i} ; \theta\left(x_{i}\right)\right) p\left(x_{i}=j\right)\right\}
$$

Thus, the intensity distribution at pixel $i$ is modeled as a Gaussian mixture, given the distortion field. Assuming that the pixel intensities are statistically independent, the probability density for the entire image, given the distortion field, is

$$
p(y \mid d)=\prod_{i \in s} p\left(y_{i} \mid d_{i}\right)
$$

Bayes' rule can be used to obtain the posterior probability of the distortion field, given the observed intensity values

$$
p(d \mid y)=\frac{p(y \mid d) p(d)}{p(y)}
$$

Where $p(y)$ is normalization constant. The prior probability density of the distortion field $p(d)$ is modeled as a Gaussian with zero mean to capture its smoothness property. The maximum a posteriori (MAP) principle can be employed to obtain the optimal estimate of the distortion field $\hat{d}$, given the observed intensity values

$$
\widehat{\mathrm{d}}=\arg \max _{\mathrm{d}} \mathrm{p}(\mathrm{d} \mid \mathrm{y})
$$

The optimum solution $\hat{d}$ satisfies the following condition

$$
\left[\frac{\partial}{\partial d_{i}} \ln p(d \mid y)\right]=0 \quad \forall_{i}
$$

Solving this equation leads to the update equations (see [12] for detail)

$$
\begin{aligned}
& w_{i j}=\frac{p\left(y_{i} \mid x_{i}, d_{i}\right) p\left(x_{i}=j\right)}{p\left(y_{i} \mid d_{i}\right)} \\
& d_{i=} \frac{[F R]_{i}}{\left[F_{\psi}^{-1} I\right]_{i}} \text {, with } \mathrm{I}=(1,1, \ldots, 1)^{T} .
\end{aligned}
$$

Here, $W_{i j}$ is the posterior probability that pixel $i$ belongs to class $j$ given the distortion field estimate, $F$ is a low-pass filter, $R$ is the mean residual in which for pixel $i$

$$
\mathrm{R}_{\mathrm{i}}=\sum_{\mathrm{j} \in \mathrm{L}} \frac{\mathrm{w}_{\mathrm{ij}}\left(\mathrm{y}_{\mathrm{i}}-\mu_{\mathrm{j}}\right)}{\sigma_{\mathrm{j}}^{2}}
$$

And $\psi$ is the mean inverse covariance, in which if otherwise.

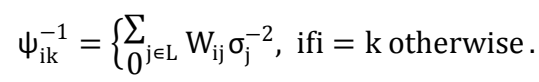

\subsection{Bayesian Criterion For Filtering Edge Information}

It is assumed that interpolated boundaries will partially overlap with the true edges found using edge detection. The probability of edges overlapping with shape-interpolated boundaries may be modelled using Bayes' probability. It is assumed that the probability of overlap at interpolated slices is greater than or equal to that at user-initialized contours. Edges are divided into edge components based on their connectivity. To retain edges with higher saliency, the edge components are sorted in descending order relative to the amount of overlap with the boundary. When the cumulative probability of overlap exceeds that obtained from user initialized contours, the remaining edge components are discarded. The Bayes' classification is thus not employed for training, but rather as a guide to how well boundaries can be defined based on edge detection.

\section{PROPOSED ALGORITHMS}

\subsection{The 3-d Cases}

The algorithm can be applied to 3-D volumes reconstructed from a sequence of parallel, closely spaced 2-D images. We assume that in such a sequence neighbouring slices resemble each other, that is, overlapping pixels in neighbouring slices tend to have the same class labels. Intensity in homogeneity field estimation is performed within each 2-D image, while the energy function in the MRF prior model involves a 3-D neighbourhood system, which includes, for each pixel in a 2D scan, the eight nearest neighbours in the same scan and the two direct neighbours in the previous and the next scan. This 3-D constraint helps to strengthen ambiguous boundaries that are easily miss allocated in 2-D processing.

\subsection{BoundaryEdge Correspondences}

Ideally, the match between boundary and edge should be oneto-one. However, deviations in the interpolated shape will not initialize $\mathrm{Bi}$ well. To prevent many-to-one snapping of boundary points, a minimum snapping-distance map is stored for every edge point. Subsequent boundary points will only be allowed to snap to the edge point if the snapping distance is less than or equal to the value in the minimum snappingdistance map. Therefore, boundary points will not arbitrarily 
snap to false edges if there are no edges to be found. During the first iteration, the search window has not been adaptively altered to match the edge proximity for the image slice. There is a possibility that a false edge will be included in the Bayesian criterion. To prevent this, an inverse weighted distance transform, $\mathrm{M}$, is multiplied to $\mathrm{Fi}, \mathrm{k}$, where $\mathrm{M}$ is a square matrix. Denoting pq $\mathrm{M}$ as an element in $\mathrm{M}$ and any two points on the $\mathrm{Bi}$ as bp and bq., pq $\mathrm{M}$ is defined in Eq. 1 .

$$
\mathrm{M}_{\mathrm{pq}}=\frac{1}{|| \mathrm{b}_{\mathrm{p}}-\mathrm{b}_{\mathrm{q}} \|++1} \ldots(1)
$$

\subsection{Local Intensity Clustering Property}

Region-based image segmentation methods typically rely on a specific region descriptor (e.g. intensity mean or a Gaussian distribution) of the intensities in each region to be segmented. However, it is difficult to give such a region descriptor for images with intensity inhomogeneities. Moreover, intensity inhomogeneities often lead to overlap between the distributions of the intensities in the regions $\Omega_{1}, \ldots, \Omega_{N}$. Therefore, it is impossible to segment these regions directly based on the pixel intensities .Nevertheless, the property of local intensities is simple, which can be effectively exploited in the formulation of our method for image segmentation with simultaneous estimation of the bias field based on the image model in (3) and the assumptions A1 and A2, we are able to derive a useful property of local intensities, which is referred to as a local intensity clustering property as described and justified below. To be specific, we consider a circular neighbourhood with a radius $\rho$ centered at each point $y \in$

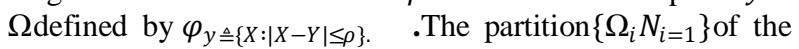
entire domain $\Omega$ induces a partition of the neighbourhood $\varphi_{\mathrm{y}}$, i.e., $\left\{\varphi_{\mathrm{y}} \cap \Omega_{\mathrm{i}}\right\} \mathrm{N}_{\mathrm{i}=1}$, , i.e., forms a partition of $\varphi_{\mathrm{y}}$. For as lowly varying bias field $\mathrm{b}$ the values $\mathrm{b}(\mathrm{X})$ for all $\mathrm{X}$ in the circular Neighbourhood $\varphi_{\mathrm{y}}$ are close to b (y), i.e. for

$$
\mathrm{b}(\mathrm{X}) \approx \mathrm{b}(\mathrm{y}) \text { for } \mathrm{X} \in \phi_{\mathrm{y}}(4)
$$

Thus, the intensities $\mathrm{b}(\mathrm{X}) \mathrm{J}(\mathrm{X})$ in each sub region $\varphi_{\mathrm{y} \cap \Omega_{\mathrm{i}}}$ are close to the constantb $(y) C_{i}$ i.e.

$$
\mathrm{b}(\mathrm{X}) \mathrm{J}(\mathrm{X}) \approx \mathrm{b}(\mathrm{y}) \mathrm{C}_{\mathrm{i}} \text { forX } \in \varphi_{\mathrm{y}} \cap \Omega_{\mathrm{i}}(5)
$$

Then, in view of the image model in (3), we have

$$
\mathrm{I}(\mathrm{X}) \approx \mathrm{b}(\mathrm{y}) \mathrm{C}_{\mathrm{i}}+\mathrm{n}(\mathrm{X}) \text { forX } \in \varphi_{\mathrm{y}} \cap \Omega_{\mathrm{i}}
$$

Where $\mathrm{n}(\mathrm{X})$ is additive zero-mean Gaussian noise Therefore, the intensities in the set

$$
\mathrm{I}_{\mathrm{y}}^{\mathrm{i}}=\left\{\mathrm{I}(\mathrm{x}): \mathrm{x} \in \varphi_{\mathrm{y}} \cap \Omega_{\mathrm{i}}\right\}
$$

Form a cluster with cluster centre $\mathrm{m}_{\mathrm{i}} \approx \mathrm{b}(\mathrm{y}) \mathrm{C}_{\mathrm{i}}$, which can be considered as samples drawn from a Gaussian distribution with mean $\mathrm{m}_{\mathrm{i}}$. ObviouslyN, the clusters $\mathrm{I}_{\mathrm{y}}^{1}, \ldots, \mathrm{I}_{\mathrm{y}}^{\mathrm{N}}$, are well-separated, with distinct cluster centers $\quad \mathrm{m}_{\mathrm{i}} \approx$ $\mathrm{b}(\mathrm{y}) \mathrm{C}_{\mathrm{i}}, \mathrm{i}=1, \ldots, \mathrm{N}$, (because the constants $\mathrm{C}_{1}, \ldots, \mathrm{C}_{\mathrm{N}}$ are distinct and the variance of the Gaussian noisen is assumed to be relatively small). This local intensity clustering property is used to formulate the proposed method for image segmentation and bias field estimation as follows.

\subsection{Energy Formulation}

The above described local intensity clustering property indicates that the intensities in the neighbourhood $\varphi_{\mathrm{y}}$ can be classified in to $\mathrm{N}$ clusters with centers $\mathrm{m}_{\mathrm{i}} \approx \mathrm{b}(\mathrm{y}) \mathrm{C}_{\mathrm{i}}, \mathrm{i}=$
$1, \ldots, \mathrm{N}$, This allows us to apply the standard K-means clustering to classify these local intensities. Specifically, for the intensities $\mathrm{bI}(\mathrm{X})$ in the neighbourhood $\varphi_{\mathrm{y}}$, the K-means algorithm is an iterative process to minimize the clustering criterion [22], which can be written in a continuous form as

$$
\mathrm{F}_{\mathrm{Y}}=\sum_{\mathrm{i}=1}^{\mathrm{N}} \int_{\varphi_{\mathrm{y}}} \mathrm{I}(\mathrm{X})-\mathrm{m}_{\mathrm{i}}^{2} \mathrm{u}_{\mathrm{i}}(\mathrm{X}) \mathrm{dx}
$$

Wherem ${ }_{\mathrm{i}}$ is the cluster center of thei - th cluster, $\mathrm{u}_{\mathrm{i}}$ is the mem $\mathrm{X} \in \Omega_{\mathrm{i}}$ and $\mathrm{u}_{\mathrm{i}}(\mathrm{X})=0 \quad$ for $\mathrm{X} \notin \Omega_{\mathrm{i}}$. Since $\mathrm{u}_{\mathrm{i}}$ is the membership function of the region $\Omega_{\mathrm{i}}$, we can rewrite $\mathrm{F}_{\mathrm{y}}$ as

$$
\mathrm{F}_{\mathrm{Y}}=\sum_{\mathrm{i}=1}^{\mathrm{N}} \int_{\Omega_{\mathrm{i}} \cap \varphi_{\mathrm{y}}} \mathrm{I}(\mathrm{X})-\mathrm{m}_{\mathrm{i}}^{2} \mathrm{dx} .
$$

In view of the clustering criterion in (7) and the approximation of the cluster center by

$\mathrm{m}_{\mathrm{i}} \approx \mathrm{b}(\mathrm{y}) \mathrm{C}_{\mathrm{i}}$, We define a clustering criterion for classifying the intensities $\operatorname{in} \varphi_{y}$ as

$$
\epsilon_{\mathrm{Y}}=\sum_{\mathrm{i}=1}^{\mathrm{N}} \int_{\Omega_{\mathrm{i}} \cap \varphi_{\mathrm{y}}} \mathrm{K}(\mathrm{y}-\mathrm{x}) \mathrm{I}(\mathrm{X})-\mathrm{b}(\mathrm{y}) \mathrm{C}_{\mathrm{i}}^{2} \mathrm{dx}
$$

Where $\mathrm{K}(\mathrm{y}-\mathrm{x})$ is introduced as a nonnegative window function, also called kernel function, such that $\mathrm{K}(\mathrm{y}-\mathrm{x})=0$ for $\mathrm{X} \notin \varphi_{\mathrm{y}}$. With the window function, the clustering criterion function $\varepsilon_{\mathrm{y}}$ can be rewritten as

$$
\epsilon_{\mathrm{Y}}=\sum_{\mathrm{i}=1}^{\mathrm{N}} \int_{\Omega_{\mathrm{I}}} \mathrm{K}(\mathrm{y}-\mathrm{x}) \mathrm{I}(\mathrm{X})-\mathrm{b}(\mathrm{y}) \mathrm{C}_{\mathrm{i}}{ }^{2} \mathrm{dx}
$$

This local clustering criterion function is a basic element in the formulation of our method.

\subsection{Multi phase Level Set Formulation}

For the case of $\mathrm{N} \geq 3$, we can use two or more level set functions $\emptyset_{1}, \ldots, \emptyset_{\mathrm{k}}$ to define $\mathrm{N}$ membership functions $\mathrm{m}_{\mathrm{i}}$ of the regions $\Omega_{\mathrm{i}}, \mathrm{i}=1, \ldots, \mathrm{N}$, , such that

$\mathrm{M}_{\mathrm{i}}\left(\emptyset_{1}(\mathrm{y}), \ldots, \emptyset_{\mathrm{k}}(\mathrm{y})\right)=\left\{\begin{array}{cc}1, & \mathrm{y} \in \Omega_{\mathrm{i}} \\ 0, & \text { else }\end{array}\right.$

For example, in the case of 3 , we use two level set functions $\emptyset_{1}$ and $\emptyset_{2}$ to define

$\mathrm{M}_{1}\left(\emptyset_{1}, \emptyset_{2}\right)=\mathrm{H}\left(\emptyset_{1}\right) \mathrm{H}\left(\emptyset_{2}\right), \mathrm{M}_{2}\left(\emptyset_{1}, \emptyset_{2}=\mathrm{H}\left(\varnothing_{1}\right)\left(1-\mathrm{H}\left(\varnothing_{2}\right)\right)\right.$

And $\mathrm{M}_{3}\left(\varnothing_{1}, \emptyset_{2}\right)=1-\mathrm{H}\left(\varnothing_{1}\right)$ to give a three-phase level set formulation of our method. For the four-phase case $\mathrm{N}=4$, the definition of $\mathrm{M}_{\mathrm{i}}$ can be defined as

$$
\begin{aligned}
\mathrm{M}_{1} & \left(\varnothing_{1}, \emptyset_{2}\right)=\mathrm{H}\left(\varnothing_{1}\right) \mathrm{H}\left(\emptyset_{2}\right), \mathrm{M}_{2}\left(\varnothing_{1}, \emptyset_{2}\right) \\
& =\mathrm{H}\left(\varnothing_{1}\right)\left(1-\mathrm{H}\left(\emptyset_{2}\right)\right), \mathrm{M}_{3}\left(\varnothing_{1}, \emptyset_{2}\right) \\
& =\left(1-\mathrm{H}\left(\varnothing_{1}\right)\right) \mathrm{H}\left(\varnothing_{2}\right),
\end{aligned}
$$$$
\text { And } \quad \mathrm{M}_{4}\left(\varnothing_{1}, \emptyset_{2}\right)=\left(1-\mathrm{H}\left(\varnothing_{1}\right)\right)\left(1-\mathrm{H}\left(\varnothing_{2}\right)\right) \text {. }
$$

(16). For the function $\Phi=\left(\emptyset_{1}, \ldots, \emptyset_{k}\right)$, This defines the regularization Terms $\mathrm{L}(\Phi)=\sum_{\mathrm{j}=1}^{\mathrm{k}} \mathrm{L}\left(\varnothing_{\mathrm{j}}\right)$ and $\mathrm{R}_{\mathrm{p}}(\Phi)=$ $\sum_{\mathrm{j}=1}^{\mathrm{k}} \mathrm{R}_{\mathrm{p}}\left(\emptyset_{\mathrm{j}}\right)$, Where $\mathrm{L}\left(\emptyset_{\mathrm{j}}\right)$ and $\mathrm{R}_{\mathrm{p}}\left(\varnothing_{\mathrm{j}}\right)$ are defined by (19) and (20) for each level set function $\emptyset_{j}$, respectively. The energy functionalF in our multiphase level set formulation is defined by

$\mathrm{F}(\Phi, \mathrm{b}, \mathrm{c}) \triangleq \varepsilon(\Phi, \mathrm{b}, \mathrm{c})+\mathrm{R}_{\mathrm{p}}(\Phi)$.

Thus, the membership functions $\mathrm{M}_{\mathrm{i}}\left(\emptyset_{1}(\mathrm{y}), \ldots, \emptyset_{\mathrm{k}}(\varnothing)\right)$ can be written as $\mathrm{M}_{\mathrm{i}}(\Phi)$. The energy $\varepsilon$ in (10) can be converted to a multiphase level set formulation 
With $e_{i}$ given by

$$
\varepsilon(\Phi, c, b)=\int \sum_{i=1}^{N} e_{i}(X) M_{i}(\Phi(X)) d x
$$

The minimization of the energy $\mathrm{F}(\Phi, \mathrm{c}, \mathrm{b})$ in (25) with respect tto the variable $\Phi=\left(\emptyset_{1}, \ldots, \emptyset_{\mathrm{k}}\right)$ can be performed by solving the following gradient flow equations:

For notational simplicity, we denote these level set functions $\emptyset_{1}, \ldots, \emptyset_{\mathrm{k}}$ by a vector valued function $=\emptyset_{1}, \ldots, \emptyset_{\mathrm{k}}$.

$$
\begin{aligned}
\frac{\partial \emptyset_{1}}{\partial \mathrm{t}}= & -\sum_{\mathrm{i}=1}^{\mathrm{N}} \frac{\partial \mathrm{M}_{\mathrm{i}}(\varnothing)}{\partial \emptyset_{1}} \mathrm{e}_{\mathrm{i}}+\mathrm{v} \partial\left(\emptyset_{1}\right) \operatorname{div}\left(\frac{\nabla \emptyset_{1}}{\left|\nabla \emptyset_{1}\right|}\right. \\
& +\mu \operatorname{div}\left(\left(\mathrm{d}_{\mathrm{p}}\left(\left|\nabla \emptyset_{1}\right|\right) \nabla \emptyset_{1}\right)\right. \\
& \cdot \\
\frac{\partial \emptyset_{\mathrm{k}}}{\partial \mathrm{t}}= & -\sum_{\mathrm{i}=1}^{\mathrm{N}} \frac{\partial \mathrm{M}_{\mathrm{i}}(\varnothing)}{\partial \emptyset_{\mathrm{k}}} \mathrm{e}_{\mathrm{i}}+\mathrm{v} \partial\left(\emptyset_{\mathrm{k}}\right) \operatorname{div}\left(\frac{\nabla \emptyset_{\mathrm{k}}}{\left|\nabla \emptyset_{\mathrm{k}}\right|}\right)
\end{aligned}
$$$$
+\mu \operatorname{div}\left(\mathrm{d}_{\mathrm{p}}\left(\left|\nabla \emptyset_{\mathrm{k}}\right|\right) \nabla \emptyset_{\mathrm{k}}\right) \text {. }
$$

\section{EXPERIMENTAL RESULTS}

To demonstrate the effectiveness of our method in simultaneous segmentation and bias field estimation, we applied it to two medical images with intensity inhomogeneities: a CT image of breast and CT image of brain, These images exhibit obvious intensity inhomogeneities. Firstly a CT original image of Breast and Brain is separately taken and the histogram images with gray level values are obtained Individually then initial contour based metric evaluation for precise segmentation result is done. CT Images are performed with 50 iterations for Checking Initial Point of the Image These images are corrupted with serious speckle noise here it is applied with a convolution of Gaussian kernel to smooth the CT image as a preprocessing step. The scale parameter of the Gaussian kernel is chosen as 2.0 for smoothing this image. The corresponding result of segmentation is bias corrected image

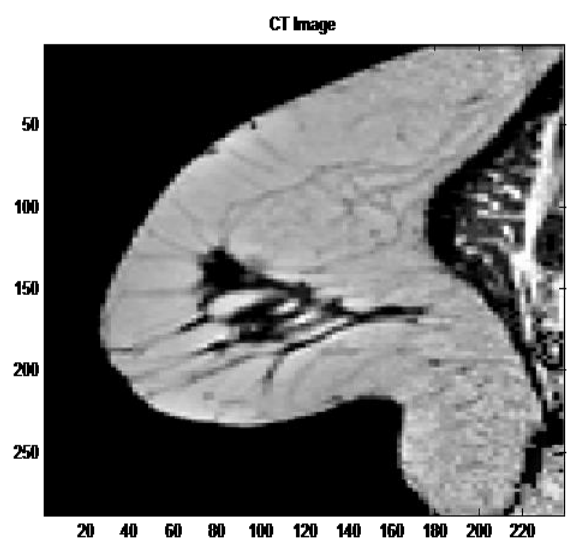

Fig 1. Shows Input Of CT Image of Breast

(Courtesy: Rainbow hospitals, India)

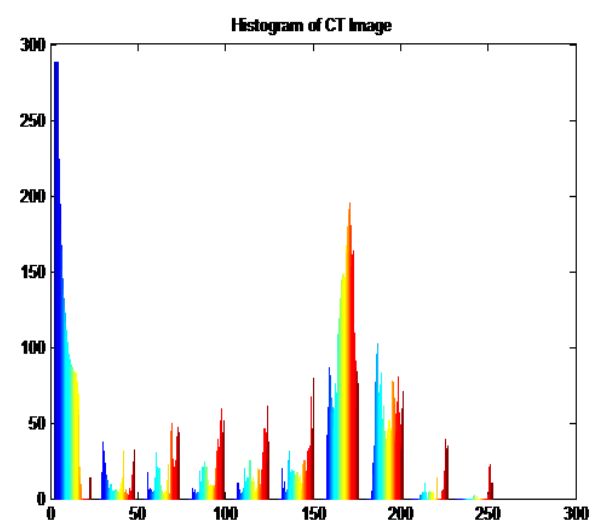

Fig 2. Histogram of above CT Image

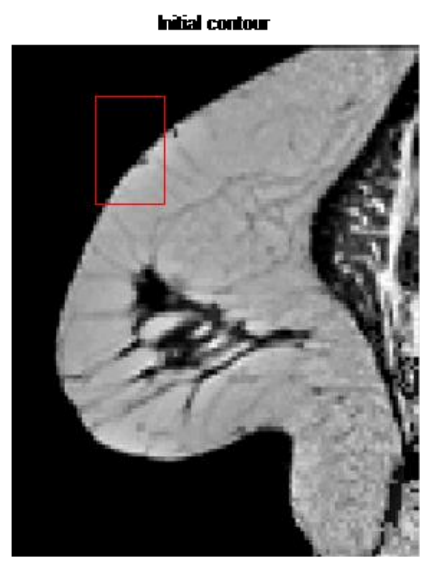

Fig 3. Intial contour of CT image

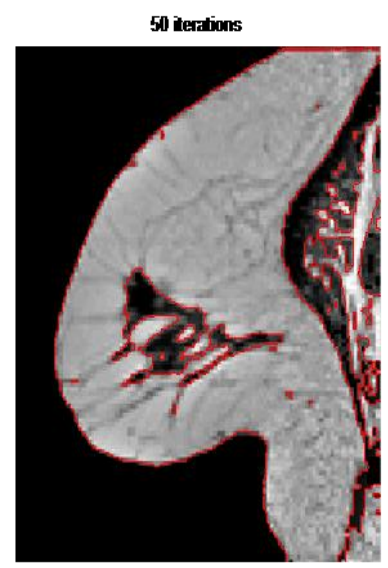

Fig 4. CT image with 50 Iter ations 


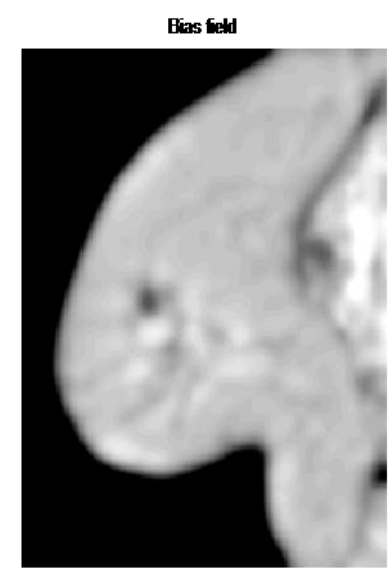

Fig 5. Blurring Of Input Imagefor Checking from Initial Point of the Image

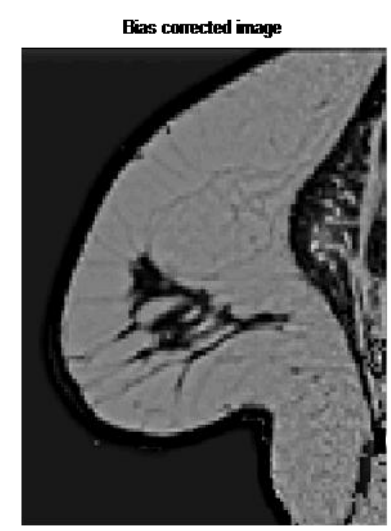

Fig 6. CT Bias Corrected Image

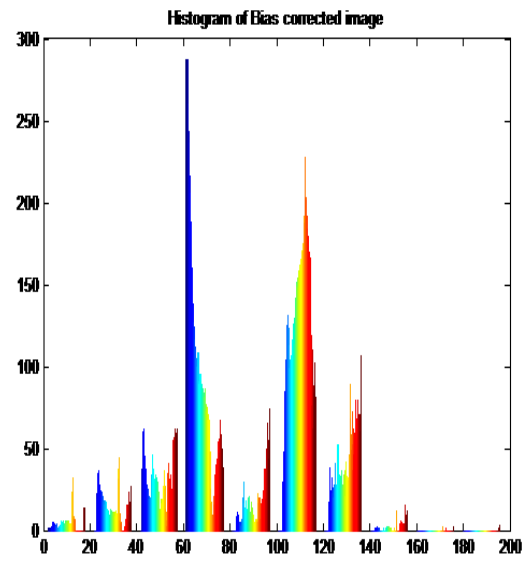

Fig 7. Image describes Histogram of Bias Corrected Image

\section{For Brain CT Images}

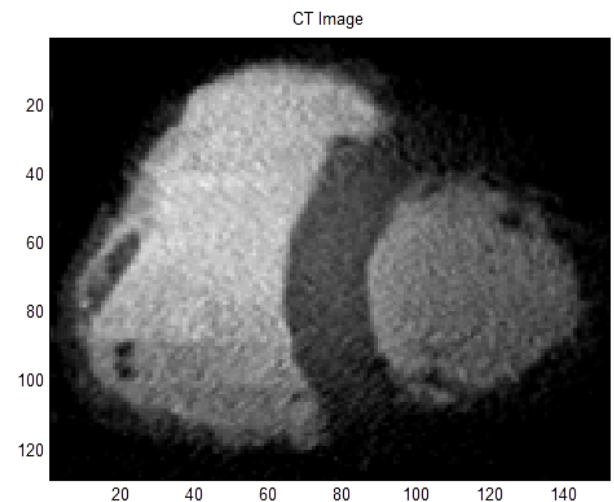

Fig 1. Shows Input Of CT Image of Brain (Courtesy: Rainbow hospitals, India)

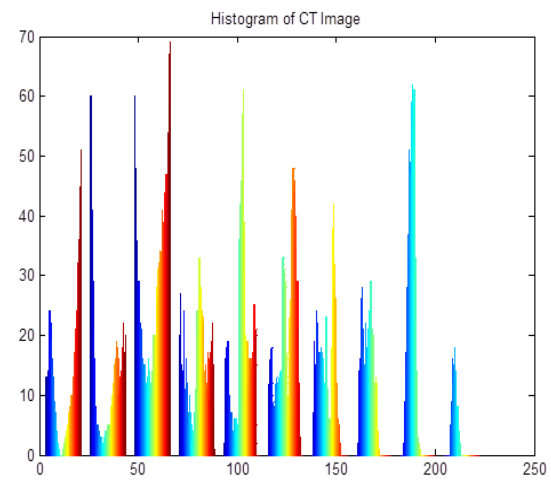

Fig 2. gives Histogram of CT Image With Density

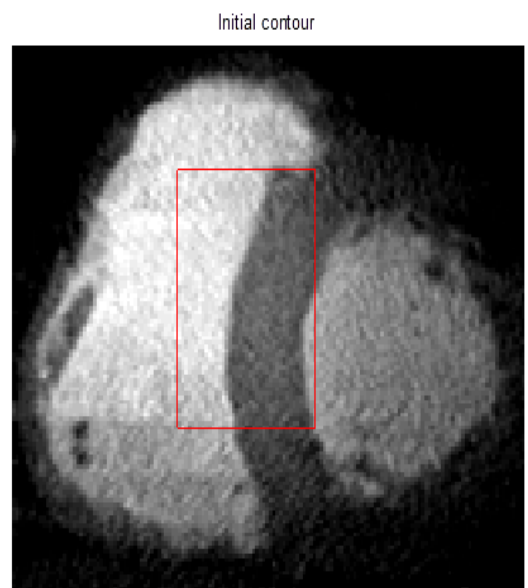

Fig 3. Iterations are Performed to the Input Image 


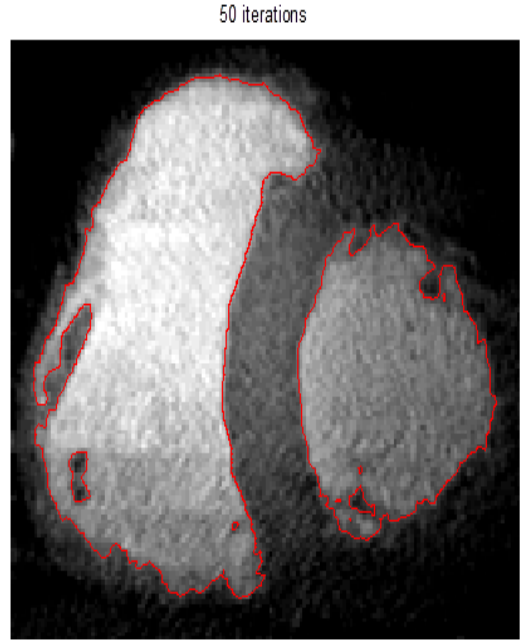

Fig 4. CT image with 50 Iterations

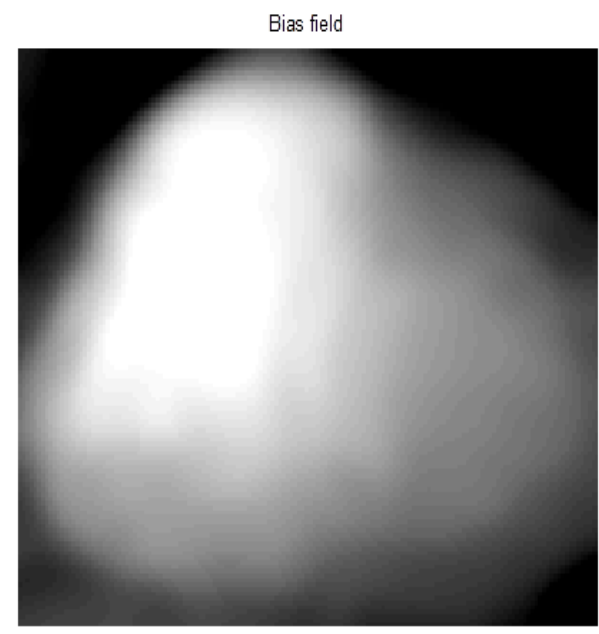

Fig 5. Blurring Of Input Imagefor Checking from Initial Point of the Image

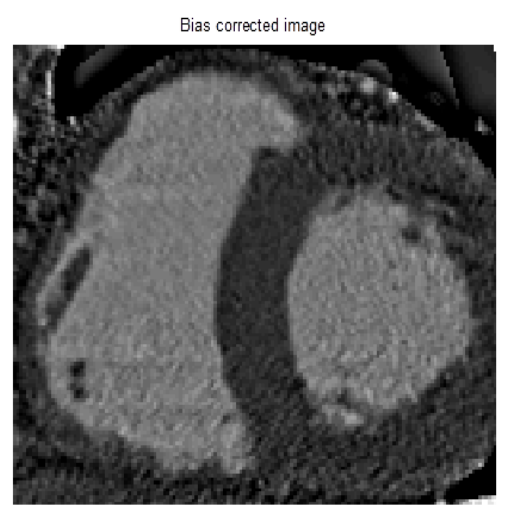

Fig 6. Input Image Of Bias Corrected Image

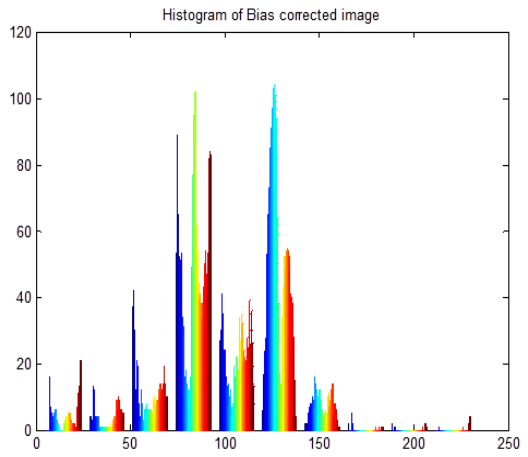

Fig 7. Image describes Histogram of Bias Corrected Image

\section{DISCUSSION}

Segmentation accuracy of our method for different initializations and different scale parameters is calculated by MSE and PSNR. It is obvious that our model produces more accurate segmentation results than other Probablistic Synthetic model. To quantitatively evaluate the accuracy, we compute the mean errors of both models for two images, On the other hand, our model is much more efficient than the PS model. This can be seen from the CPU times consumed by different models for the two images is more in this experiment, our model is remarkably faster than the PS model with an average speed-up factor in our implementation. The CPU times in this experiment were recorded in running our Matlab programs on a Lenovo ThinkPad notebook with Intel (R) Core (TM) 2 Duo CPU, $2.40 \mathrm{GHz}, 2$ GB RAM, with Matlab 7.4 on Windows Vista.

Table 1.1. Tabulation of Quantitative Measures

\begin{tabular}{|c|l|l|}
\hline $\begin{array}{c}\text { Type of CT } \\
\text { Image }\end{array}$ & PSNR Ratio & $\begin{array}{c}\text { Mean Square } \\
\text { Error }\end{array}$ \\
\hline Breast & 11.6767 & $2.6557 \mathrm{e}+003$ \\
\hline Brain & 14.2813 & $2.0117 \mathrm{e}+003$ \\
\hline
\end{tabular}

\section{CONCLUSION}

This work presents a variational level set framework for segmentation and bias correction of images with intensity inhomogeneities. Based on a generally accepted model of images with intensity inhomogeneities and a derived local intensity clustering property, the work defines an energy of the level set functions that represent a partition of the image domain and a bias field that accounts for the intensity inhomogeneity. Segmentation and bias field estimation is given in the results of Breast cancer and tumour detection which is possible done. There Are therefore jointly performed by minimizing the proposed energy functional. The slowly varying property of the bias field derived from the proposed energy is naturally ensured by the data term in our variational framework, without the need to impose an explicit smoothing term on the bias field. The proposed method is much more robust to initialization than the piecewise smooth model. Experimental results have demonstrated superior performance of our method in terms of accuracy, efficiency, and robustness. 


\section{ACKNOWLEDGMENT}

The work was supported under Research grants of R.P.S A.I.C.T.E, New Delhi and Annamacharya Institute of Technology \& Sciences, Rajampet.

\section{REFERENCES}

[1] Olabarriaga,S.D. and Smeulders, A.W.M., "Interaction in the Segmentation of Medical Images: A Survey",Med. Image Analysis, 5: 127-142, 2001.

[2] PN.T. Wells and M.Halliwell, "Speckle in ultrasonic imaging," Ultrasonic's, vol. 19, pp. 225-229, 1981.

[3] R. F. Wagner, S. W. Smith, J. M. Sandrik, and H. Lopez, "Statistics of speckle in ultrasound B-scans," IEEE Trans. Sonics Ultrasonic's, vol. SU-30, pp. 156-163, Mar. 1983.

[4] J. C. Bamber and C. Daft, "Adaptiv filtering for reduction of speckle in ultrasonic pulse-echo images," Ultrasonic Image., pp. 41-44, January 1986.

[5] G. Castellini, D. Lamate, L. Mascotti, E. Monnini, and S. Rocchi, "An adaptive Kalman filter for speckle reduction in ultrasound images," J. Nucl. Med. Appl. Sci., vol. 32, no. 3 , p. 213,1988 .

[6] D. Kaplan and Q. Ma, "On the statistical characteristics of log-compressed Rayleigh signals: Theoretical formulation and experimental results," J. Acoust. Soc. Amer., vol. 95, no. 3, pp. 1396-1400, 1994.

[7] V. Dutt and J. F. Greenleaf, "Adaptive speckle reduction filter for log-compressed B-scan images," IEEE Trans. Med. Image., vol. 15, pp. 802-813, Dec. 1996.

[8] A. N. Evans and M. S. Nixon, "Biased motion-adaptive temporal filtering for speckle-reduction in echocardiography," IEEE Trans. Med. Image., vol. 15, pp. 39-50, Feb. 1996.

[9] T. Chan and L. Vese, "Active contours without edges," IEEE Trans. Image. Process. vol. 10, no. 2, pp. 266-277, Feb. 2001.

[10] C. Li, C. Kao, J. C. Gore, and Z. Ding, "Minimization of region-scalable fitting energy for image segmentation," IEEE Trans. Image Process., vol. 17, no. 10, pp. 19401949, Oct. 2008.

[11] V. Caselles, R. Kimmel, and G. Sapiro, "Geodesic active contours," Int. J. Comput. Vis., vol. 22, no. 1, pp. 61-79, Feb. 1997.

[12] S. Kichenassamy, A. Kumar, P. Olver, A. Tannenbaum, and A. Yezzi, "Gradient flows and geometric active contour models," in Proc. 5th Int. Conf. Comput. Vis., 1995 , pp. 810-815.

[13] R. Kimmel, A. Amir, and A. Bruckstein, "Finding shortest paths on surfaces using level set propagation," IEEE Trans. Pattern Anal. Mach. Intell., vol. 17, no. 6, pp. 635-640, Jun. 1995.

[14] R. Malladi, J. A. Sethian, and B. C.Vemuri, "Shape modeling with front propagation: A level set approach," IEEE Trans. Pattern Anal. Mach. Intell., vol. 17, no. 2, pp. 158-175, Feb. 1995.

[15] R. Ronfard, "Region-based strategies for active contour models," Int. J. Comput. Vis., vol. 13, no. 2, pp. 229251, Oct. 1994
[16] C. Samson, L. Blanc-Feraud, G. Aubert, and J. Zerubia, "A variational model for image classification and restoration," IEEE Trans. Pattern Anal. Mach. Intell., vol. 22, no. 5, pp. 460-472, May 2000.

[17] S. Theodoridis and K.Koutroumbas, Pattern Recognition. New York: Academic, 2003.

[18] A. Tsai, A. Yezzi, and A. S.Willsky, "Curve evolution implementation of the Mumford-Shah functional for image segmentation, de noising, interpolation, and magnification," IEEE Trans. Image Process., vol. 10, no. 8, pp. 1169-1186, Aug. 2001.

[19] A. Vasilevskiy and K. Siddiqi, "Flux-maximizing geometric flows," IEEE Trans. Pattern Anal. Mach. Intell., vol. 24, no. 12, pp. 1565-1578, Dec. 2002.

[20] L. Vese and T. Chan, "A multiphase level set framework for image segmentation using the Mumford and Shah model," Int. J. Comput. Vis., vol. 50, no. 3, pp. 271-293, Dec. 2002.

[21] N. Paragios and R. Deriche, "Geodesic active regions and level set methods for supervised texture segmentation," Int. J. Comput. Vis., vol. 46, no. 3, pp. 223-247, Feb. 2002.

[22] S. Theodoridis and K.Koutroumbas, Pattern Recognition. NewYork:Academic, 2003.

[23] S. Asif Hussain and Dr. M.N. Giri Prasad, Dr, D. Satya Narayana "3d Medical image modeling with dynamic image reconstruction "in IJERT Vol 5, No.3 (2012) PP. 213-217

\section{AUTHOR'S PROFILE}

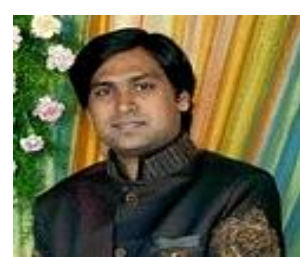

Asif Hussain. Shaik received B.Tech \& M.Tech Degree in Electronics \& Communication Engg. from JNTUniversity, Hyderabad, India. He is currently working towards $\mathrm{PhD}$ Degree in Biomedical Image Processing at JNTU University, Annantapur, India. Presently he is with Annamacharya Institute of Technology \& Sciences, Rajampet, A.P.,India.

$\mathrm{He}$ is working as Assistant Professor in Dept. of ECE. He presented many research papers in National \& International Conferences\& journals. $\mathrm{He}$ is a member of Professional societies like ISTE (India), BMESI (India), IACSIT (Singapore), IAENG (Hongkong) and WASE (Hongkong).His research interests include Signal Processing, Time Series Analysis and Image Processing.

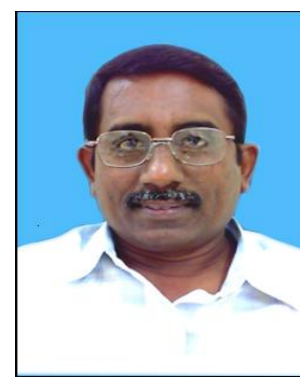

Dr. M. N. Giri Prasad received B.Tech Degree in Electronics \& Communication Engineering from JNT University, Hyderabad, India. He received his M.Tech Degree from S.V.University, Tirupathi, India. He has received $\mathrm{PhD}$ in Biomedical Signal Processing from JNT University, Hyderabad, India. 
At Present he is Professor in Dept. of ECE at JNTUCE, Anantapur, A.P., India. He also worked as Principal at JNTUCE, Pulivendula, A.P., India. He has more than 20 years of teaching experience. He has more than 30 publications in standard International/technical Journals. His research interests include Biomedical Signal Processing, Antennas and Image Processing.

$\mathrm{He}$ is a member of Professional societies like IE (India), NAFEN (India), ISTE (India), IACSIT (Singapore),CSTA(USA) and IAENG (Hongkong). He is reviewer of Engineers Australia Technical Journals, Australia, of International Journal of Computer Science and Information Security (IJCSIS), Pittsburgh, USA, International Journal of Electrical, Electronics and Computer Systems (IJEECS), USA and International Journal of Algorithm, of AWEM Group, USA. He is reviewer of National/International Conferences in India and IEEE Sponsored International Conferences held in Singapore and China. He chaired many National/International conferences in India. He is BOS member of reputed engineering colleges in Andhra Pradesh.

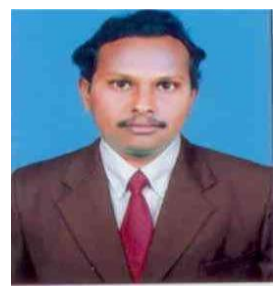

Dr.D.Satyanarayana, Professor in Electronics \& Communication Engineering, having 18 years teaching experience, has been graduate $\mathrm{d}$ from Bharatiar University, Coimbatore in the faculty of Electronics \& Communication Engineering in 1992. Later he has obtained his Master's Degree in Digital Systems and Computer Electronics from Jawaharlal Nehru Technology University, Hyderabad in the year 1998.

He was awarded a Doctorate for his work in Signal Processing by J.N.T. University, Hyderabad in the year 2009. At present he is heading the department of ECE at RGMEC. He is very actively involved in Research work and presented 10 Research papers on different topics in the field of Electronics. He is a member of Professional societies like ISTE (India), IETE (India) and IEEE. In his total Endeavour as a teacher, he is known for his friendliness with the students and his colleagues. He is a man of simplicity, sincerity and hard working. 